OPEN ACCESS

Edited by:

Antonio Riva,

Foundation for Liver Research,

United Kingdom

Reviewed by:

Frank Tacke

Charité-Universitätsmedizin

Berlin, Germany

Albrecht Pliper,

University Hospital Frankfurt, Germany

*Correspondence:

Divya P. Kumar

divyapkumar@jssuni.edu.in

Specialty section:

This article was submitted to

Inflammation,

a section of the journal

Frontiers in Immunology

Received: 09 November 2020

Accepted: 17 December 2020

Published: 02 February 2021

Citation:

Srinivas AN, Suresh D,

Santhekadur PK, Suvarna $D$ and

Kumar DP (2021) Extracellular Vesicles as Inflammatory Drivers in NAFLD.

Front. Immunol. 11:627424. doi: 10.3389/fimmu.2020.627424

\section{Extracellular Vesicles as Inflammatory Drivers in NAFLD}

\author{
Akshatha N. Srinivas ${ }^{1}$, Diwakar Suresh ${ }^{1}$, Prasanna K. Santhekadur ${ }^{1}$, Deepak Suvarna ${ }^{2}$ \\ and Divya P. Kumar ${ }^{1 *}$ \\ ${ }^{1}$ Department of Biochemistry, CEMR, JSS Medical College, JSS Academy of Higher Education and Research, Mysuru, India, \\ 2 Department of Gastroenterology, JSS Medical College and Hospital, JSS Academy of Higher Education and Research, \\ Mysuru, India
}

Non-alcoholic fatty liver disease (NAFLD) is a highly prevalent chronic liver disease in most parts of the world affecting one-third of the western population and a growing cause for end-stage liver diseases such as hepatocellular carcinoma (HCC). Majorly driven by obesity and diabetes mellitus, NAFLD is more of a multifactorial disease affected by extrahepatic organ crosstalk. Non-alcoholic fatty liver (NAFL) progressed to non-alcoholic steatohepatitis (NASH) predisposes multiple complications such as fibrosis, cirrhosis, and HCC. Although the complete pathogenic mechanisms of this disease are not understood, inflammation is considered as a key driver to the onset of NASH. Lipotoxicity, inflammatory cytokines, chemokines, and intestinal dysbiosis trigger both hepatic and systemic inflammatory cascades simultaneously activating immune responses. Over a few years, extracellular vesicles studied extensively concerning the pathobiology of NAFLD indicated it as a key modulator in the setting of immune-mediated inflammation. Exosomes and microvesicles, the two main types of extracellular vesicles are secreted by an array of most mammalian cells, which are involved mainly in cell-cell communication that are unique to cell type. Various bioactive cargoes containing extracellular vesicles derived from both hepatic and extrahepatic milieu showed critical implications in driving steatosis to NASH reaffirming inflammation as the primary contributor to the whole process. In this minireview, we provide brief insights into the inflammatory mediators of NASH with special emphasis on extracellular vesicles that acts as drivers of inflammation in NAFLD.

Keywords: exosomes, microvesicles, nonalcoholic fatty liver disease, inflammation, cytokines, immune system

\section{INTRODUCTION}

Non-alcoholic fatty liver disease (NAFLD), a multisystem disease comprises a spectrum of histological conditions ranging from simple steatosis to non-alcoholic steatohepatitis (NASH), fibrosis, cirrhosis, and hepatocellular carcinoma (HCC) $(1,2)$. NAFLD linked with several extrahepatic chronic complications in particular diabetes mellitus and cardiovascular diseases support the fact that it is a hepatic manifestation of metabolic syndrome (3). With the growing burden and rising prevalence over the last decade, studies estimate that over $25 \%$ of the population is affected by NAFLD globally with obesity and type 2 diabetes mellitus (T2DM) being the major etiologies $(4,5)$. This number is predicted to increase by $2-3$ folds by 2030 mainly contributed by sedentary lifestyles 
and high-calorie diet intake $(6,7)$. Although steatosis is benign, $7 \%-30 \%$ of affected patients develop NASH over time, which is the major risk factor for cirrhosis and HCC. NAFLD being the common cause of the chronic liver disease is also becoming the leading cause of liver transplantation worldwide $(8,9)$.

The pathogenic mechanisms of development and progression of the disease have evolved from simple "two-hit" to complex "multiple-hit" hypothesis in which various factors and interorgan crosstalk synergistically acts providing solid evidence to NAFLD as a multifactorial condition (10). The fatty liver (NAFL) progresses to NASH through various insults causing hepatic injury, ballooning, cell death, inflammation, and consequently progresses to fibrosis (11). Several factors like lipotoxicity, immune response, and gut microbial dysbiosis drive the inflammation in NASH progression (12). Recently, extracellular vesicles involved in cell-cell communications have gained prime attention in the pathogenesis of the disease which is also a promising candidate for biomarkers and therapy in the scenario of limited diagnostic and treatment options in NAFLD $(13,14)$. Exosomes and microvesicles, the two main populations of extracellular vesicles differing in their size and biogenesis contribute to the pro-inflammatory responses that are critical for the progression of NASH (14). Furthermore, there is growing evidence that extracellular vesicles are also the major contributors in initiating inflammatory responses in liver diseases such as viral hepatitis, alcoholic fatty liver disease, and liver cancer (14-16). This review encapsulates the mechanistic role of inflammatory drivers in NAFLD progression, especially focusing on extracellular vesicles in this cellular crosstalk.

\section{LIPOTOXICITY}

The intricacy of NAFLD pathobiology is mainly because of multiple parallel hits. Although the root cause for the disease onset is imbalanced fatty acid metabolism, peripheral lipolysis, and elevated de novo lipogenesis (DNL), the accumulation of specific toxic lipid intermediates are the main culprits in driving disease progression $(17,18)$. In addition to non-esterified fatty acids, diacylglycerols, lysophosphatidylcholine, ceramides, and free cholesterol falls under the category of lipotoxic species, which are known to be the critical mediators of inflammation in NAFLD $(17,19)$. Of note, studies by Yamaguchi et al., have provided experimental evidence that the "quality" of hepatic lipid accumulation plays a key role as opposed to "quantity" of lipids with the risk factor in disease progression and also emphasizes the protective role of triglyceride formation against toxic lipids (20). Along similar lines, while the saturated and trans fatty acids (SFA and TFA) are known to be proinflammatory, in vitro and in vivo models have demonstrated that monounsaturated fatty acids (MUFA) and polyunsaturated fatty acids (PUFA) are cytoprotective aiding in the resolution of inflammation (21-23).

Lipotoxicity leading to mitochondrial and endoplasmic reticulum dysfunction, hepatocellular injury is coupled with activation of apoptotic pathways and inflammasome triggering chronic inflammatory cascades in NASH progression (24-27). Hepatic cell injury is mainly caused by the activation of toll-like receptor 4 (TLR-4), which stimulates TNF- $\alpha$ production via the c-Jun N-terminal kinase (JNK) and nuclear factor kappa B (NF$\kappa \mathrm{B})$ signaling pathways (28). Lipotoxicity causes cell death and acts as a driver of inflammation by activating tumor necrosis factor (TNF)-related apoptosis-inducing ligand receptor 2 (TRAIL-2) and Fas signaling (29). Furthermore, studies by Puri et al., have demonstrated endoplasmic reticulum (ER) as a major contributor in the pathogenesis of NAFLD (24) along with all other studies that underscored the role of oxidative stress (30). Lipotoxic species in extra-hepatic compartments also have predominant pathogenic effects orchestrating metabolic syndrome, gut dysbiosis with NASH progression (31). Recent studies have highlighted the involvement of non-coding RNAs as a mediator of lipotoxic responses especially in TLRs activated by toxic lipids such as palmitate that modulate cellular functions promoting inflammation $(32,33)$. The extracellular vesicles that are released by the lipotoxic hepatocytes and its role in amplifying inflammation are reviewed in the corresponding section below.

\section{CYTOKINES AND CHEMOKINES}

Relatively innocuous and reversible NAFL that progresses to $\mathrm{NASH}$ with the development of hepatic chronic inflammation is characterized by activation of the immune system and several inflammatory cells (34). The activation of immune cells such as macrophages (Kupffer cells), neutrophils, dendritic cells, Thelper cells, B cells, or cytotoxic T cells produces cytokines or chemokines that instigate and drive inflammatory infiltrates in NASH (35). Customary cytokines associated with NAFLD are tumor necrosis factor $\alpha$ (TNF $\alpha$ ), transforming growth factor $\beta 1$ (TGF- $\beta 1$ ), various interleukins (IL-6, IL-8, IL-10), and adipocytokines (36). TNF $\alpha$, released by most immune cells including macrophages, is a first described and one of the major cytokines implicated in NASH evolution (37). Interleukin family cytokines such as IL-6, IL-1 $\beta$, and IL-18 regulate steatohepatitis progression through hepatic apoptosis, insulin resistance, and induce inflammation via activating NF- $\kappa$ B pathways (38-40). Studies by Den Boer et al., and Cintra et al., have demonstrated that deficiency of IL-10, an anti-inflammatory cytokine has a significant role in steatosis and also stimulates the release of proinflammatory cytokines such as TNF $\alpha$, IL-6, and IL-1 $\beta$ (41, 42). Similarly, adiponectin released from white adipose tissue attenuated inflammation and fibrosis via IL-10 by blocking NF$\kappa \mathrm{B}$ inflammatory cascade (43). Besides, chemokines such as monocyte chemoattractant protein 1 (MCP-1)/CCL2, RANTES/CCL5, and other CXC chemokines have gained special importance in the progression of NAFL to NASH by regulating insulin resistance and steatosis via the inflammatory crosstalk between liver and adipose tissue (44). Thus, the inflammatory mediators such as cytokines and chemokines (and its receptors) are considered as attractive therapeutic targets in the treatment of NAFLD (45). 


\section{GUT MICROBIOTA}

The gut-liver organ crosstalk has been proposed to play a key role in NAFLD pathobiology (23). The gut microbiota exerting beneficial effects over host homeostasis turns out to cause liver damage when there is a perturbation of intestinal microbiota termed as dysbiosis (46). It is reported that various environmental factors such as drugs, diet, host immune system, and the state of intestinal mucosa cause dysbiosis resulting in the formation of leaky gut (47). The increased gut permeability, excess production of endotoxins and ethanol, altered bile acid composition results in the translocation of gut-derived inflammatory products into the liver via portal vein (48). Thus intestinal dysbiosis causes systemic inflammation in NASH (49).

Studies in germ-free animal models have revealed the significance of the intestinal microbiome and its role in NAFLD development $(50,51)$. In addition, studies have shown the host-specific effects of microbial metabolome due to varying individuals' microbiota, diet, lifestyle, and perinatal milieu (52). Lipopolysaccharides (LPS) activate TLR4 and TLR9 signaling on kupffer cells and induce the production of proinflammatory cytokines and chemokines (53). Furthermore, the inflammasomes (NRLP3 and NRLP6) that recognize damageassociated molecular patterns (DAMPs) derived from toxic lipid species and pathogen-associated molecular proteins (PAMPs) derived from gut microbial products activate the production of IL-18 and IL-1 $\beta$ and promotes liver inflammation $(11,54)$. Henceforth, it is evident that gut microbiota acts as a driver of inflammation in NAFLD $(47,52)$.

\section{EXTRACELLULAR VESICLES: INFLAMMATORY CROSSTALK IN NAFLD}

Extracellular vesicles (EVs) are non-nucleated, lipid-bound particles secreted by the cells into the extracellular space (55). The study and significance of extracellular vesicles became evident after the work of Harding et al., and Pan and Johnstone, the two independent groups in $1983(56,57)$. Over the last decade, the field of extracellular vesicles has gained prime importance with the discovery of its role as novel mediators in cell signaling. However, the increased scientific publications and dilemma created with the misnomer of extracellular vesicles led to the formation of "The International Society for Extracellular Vesicles" (ISEV). According to the Minimal Information for Studies of Extracellular Vesicles (MISEV) guidelines of 2014 proposed by this society, extracellular vesicles has two subtypes, such as exosomes (30-150 $\mathrm{nm}$ in diameter) that are endosome derived and microvesicles (50-1000 nm) or ectosomes, that are derived from the plasma membrane (58). While there exist substantial overlap/no specific protein markers and lack of standardized isolation and analysis methods of extracellular vesicles, the other subtypes can be referred to a) physical characteristics or density of EVs (small EVs, medium/large EVs with specified range); b) biochemical composition (CD81 positive-, CD26 positive-, CD63 positive- EVs); or c) based on the cell of origin (podocyte EVs, Oncosomes, apoptotic bodies) $(59,60)$.

It is interesting to note that EVs are highly heterogeneous concerning their composition, location, and function as indicated by their parental cells (61). While studies have shown that endosomal sorting complex required for transport (ESCRT) - dependent or-independent machinery is involved in the selection, formation, and grouping of cargos in exosomes (62, 63), the route of microvesicles formation requires cytoskeleton components, molecular motors, and fusion machinery (64). EVs implicated in cell-cell communication are selectively packaged and are enriched with different types of proteins (heat shock proteins-Hsp70, Hsp90; tetraspanins-CD81, CD63, CD9; cytoskeletal proteins-tubulin and actin and other proteins, such as Tsg101, GTPases, Annexins, and cytosolic proteins), lipids (cholesterol, ceramides, sphingomyelin, phosphatidylcholine, and phosphatidylserine), RNAs (messenger RNA, micro RNA, ribosomal RNA, non-coding RNA), and DNA (mitochondrial DNA) as their cargo and can transfer them to other cell types (59, 64). The cargo transfer regulates various cellular activities ranging from gene expression to metabolism (59). Of note, the number of EVs produced and the cargos loaded into these extracellular vesicles depends on the state (physiological or pathological) and microenvironment of the donor cells (65). In this mini-review, we will use the generic term extracellular vesicles (EVs) and decode its role as drivers of inflammation in NAFLD.

The multicellular organ liver with different cell types creates a need for effective intercellular communication to maintain homeostasis. Thus, the cell types of the liver- hepatocytes, kupffer cells, hepatic stellate cells, and natural killer (NK) cells secrete as well as receive the cargos from a plethora of extracellular vesicles (66). The EVs released in basal conditions to meet the normal cell-cell communication seem to increase during various liver diseases including fatty liver diseases (alcoholic fatty liver disease and non-alcoholic fatty liver disease), drug-induced liver injury, viral hepatitis, and liver cancer (16). Studies in high-fat-induced mouse and rat models have shown a significant increase in plasma EV levels, which increase with the NAFLD spectrum (67). It is speculated that hepatocellular stress or injury caused due to lipotoxic lipid species play important role in the elevated release of circulating EVs in a time- and concentration-dependent manner (68). Thus, the released EVs account for processes in NAFLD pathogenesis by promoting inflammation, immune modulation, fibrosis, and angiogenesis $(15,69)$. Besides hepatocytes, nonparenchymal cellderived and extra-hepatic origin EVs are also involved in accelerating the disease progression as there exist inter-cellular and inter-organ crosstalk in NAFLD (Figure 1). Heterogeneous cargoes with antigen specificity have been identified in these EVs whose actions are dynamic and correlates with histopathological changes in disease $(70,71)$. In this mini-review, we will discuss mainly the role of EVs and the molecular mechanisms involved in amplifying inflammation by EVs in NAFLD. 


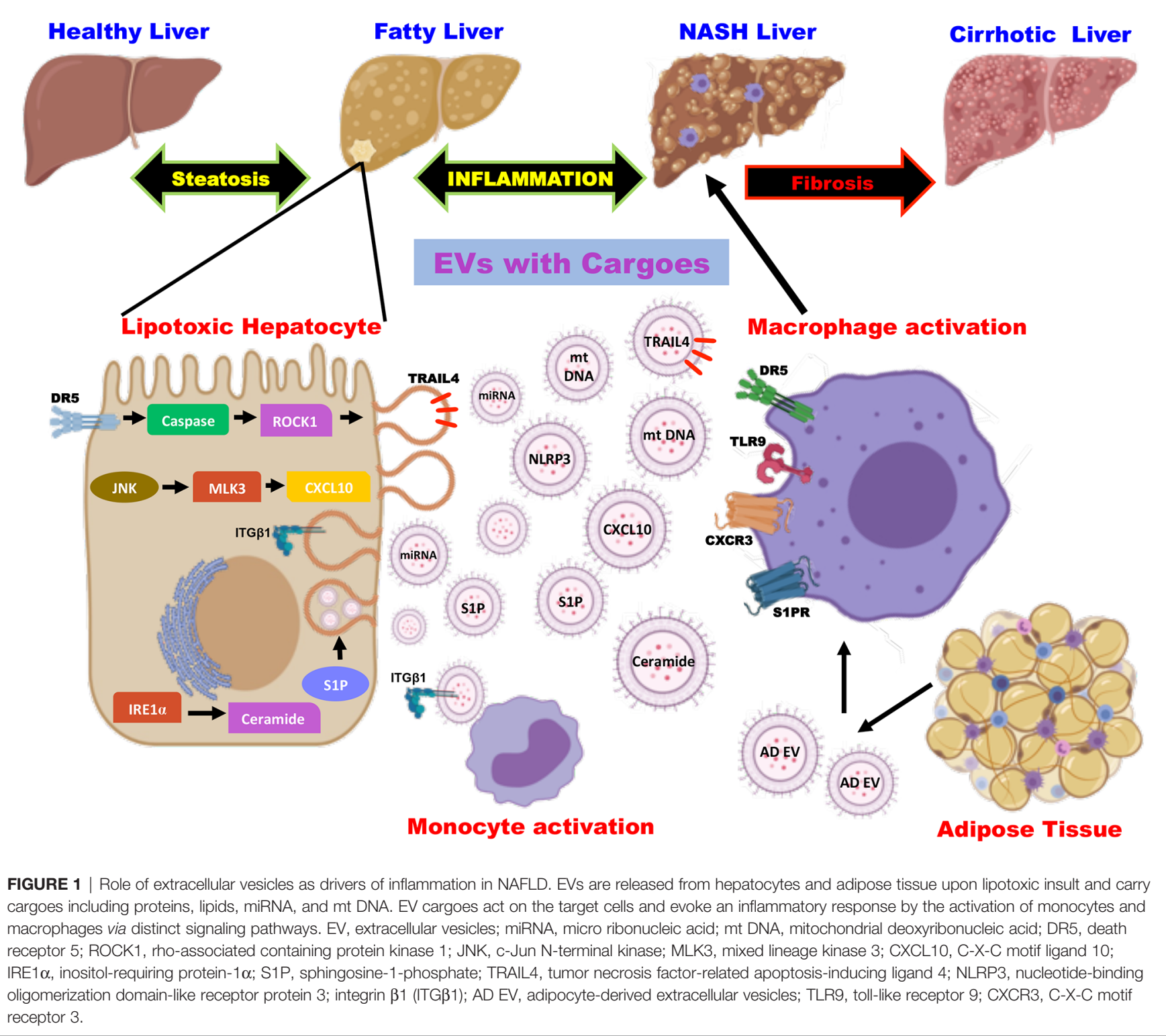

Hepatocytes are the major cell types of the liver comprising of $80 \%$ of liver volume and hence the hepatocyte-derived EVs play a critical role in the pathogenesis of NAFLD $(15,72)$. Studies on NASH in human subjects and mouse models have demonstrated that hepatocytes are the major contributors to the increased levels of circulating EVs (69). The mechanistic studies have shown that EVs released from the hepatocytes carrying different types of cargoes act in the local microenvironment via distinct cell signaling pathways (Figure 1). Kakazu et al., showed that lipotoxic insult to hepatocytes upon treatment with palmitate induces ER stress and activates inositolrequiring protein-1 $\alpha$ (IRE1 $\alpha$ ), protein kinase R-like ER kinase (PERK), and activating transcription factor $6 \alpha$ (ATF6 $\alpha$ ) leading to the synthesis of ceramides. These EVs carrying ceramides and its metabolite sphingosine-1-phosphate (S1P) activates macrophages by binding to its receptor (73). Along similar lines, lipotoxicity caused by lysophosphatidylcholine (LPC), a metabolite of palmitate induces the production of EVs loaded with tumor necrosis factor-related apoptosis-inducing ligand (TRAIL) in a death receptor 5 (DR5), caspase and rhoassociated containing protein kinase 1 (ROCK1)- dependent manner $(74,75)$. The TRAIL embedded in the EVs further activates DR5 on the macrophages leading to the production of proinflammatory cytokines such as IL-6 and IL-1 $\beta$ (76). Likewise, inhibition of TRAIL signaling (inhibition of caspase or ROCK1) has been confirmed to be an interesting therapeutic option by improving inflammation, cell injury, and fibrosis (77). Furthermore, Ibrahim et al., have shown that LPC also induces the production of EVs containing $\mathrm{C}-\mathrm{X}-\mathrm{C}$ motif ligand 10 (CXCL10) that activates macrophage chemotaxis in a c-Jun Nterminal kinase (JNK) and mixed lineage kinase 3 (MLK3)dependent mechanism by both in vitro and in vivo experiments 
using MLK3 knock out mouse primary hepatocytes and MLK3 knock out mice, respectively (78).

Studies have also shown that the EVs containing bioactive cargoes like mitochondrial DNA in mice and humans that are implicated in macrophage activation via toll-like receptor 9 (TLR-9) serves as an inflammatory signal (79). Cannito et al., have demonstrated that lipotoxic hepatocytes activate the multiprotein platform complex nucleotide-binding oligomerization domain-like receptor protein 3 (NLRP3) inflammasome leading to the production of proinflammatory cytokines (80). On the other hand, cell adhesion molecule termed integrin $\beta 1$ (ITG $\beta 1$ ) that is released as cargo from EVs of lipotoxic hepatocytes are known to mediate monocyte adhesion to liver sinusoidal endothelial cells and promote inflammation in a mouse model of NASH (81). Recent studies by Jiang et al., described that EVs released by steatotic hepatocytes express high levels of microRNA-1 that mediate proinflammatory signals in endothelial cells by NF- $\mathrm{\kappa B}$ activation and Kruppel-like factor 4 (KLF4) downregulation. This seminal work highlights the potential role of hepatocyte-derived EVs in endothelial inflammation leading to atherosclerosis (82). Similarly, the miRNA- let-7e-5p, a component of the EV derived from the hepatocytes is known to enhance lipid accumulation in the adipocytes underscoring the inter organ communication in the pathogenesis of NAFLD (83). It is also noteworthy to mention that EVs containing microRNA 192-5p derived from lipotoxic hepatocytes activate macrophages and increase IL- 6 and TNF- $\alpha$ expression and promote inflammation in NAFLD (84).

In addition to promoting inflammation, the EVs released from lipotoxic hepatocytes are also enriched with vanin-1 $(\mathrm{VNN})$, which showed a potent role in angiogenic and fibrogenic signaling pathways in endothelial cells and hepatic stellate cells (HSCs), respectively (85). Various micro RNAs (miR) are found to be an important component of extracellular vesicles leading to inflammation and fibrosis (69). Studies by Lee et al., have demonstrated that upon treatment of lipotoxic palmitate, hepatocytes show elevated levels of EVs (5-fold increase when compared to controls) and microRNAs (miRNA-122 and miRNA-192) that are implicated in driving steatohepatitis to fibrosis by upregulating the expression of genes such as $\alpha$ smooth muscle actin ( $\alpha$ SMA), collagen type 1 alpha 1 (coll $\alpha 1$ ), and transforming growth factor $\beta$ (TGF $\beta$ ) in HSC (86). Consistent with this study, elevated levels of miR-122 and miR192 were observed in mice treated with CDAA (choline-deficient L-amino acid, a diet that causes steatohepatitis) when compared to control diet-fed mice (67) and there also exists a significant positive correlation of these miRNAs with liver enzymes and disease severity in NAFLD patients (87).

As mentioned previously, in NASH, EV-mediated intercellular and interorgan crosstalk represents central events in the progression of the disease. Besides hepatocyte-derived EVs, the EVs derived from other non-parenchymal cells also play a pivotal role in the pathogenesis of NASH $(69,88)$. While EVs from cholangiocytes are known to induce angiogenic signaling in liver sinusoidal endothelial cells, studies by Wang et al., have reported that EVs derived from the endothelial cells contribute to liver fibrosis via sphingosine kinase 1 (SK1) $(89,86)$. Furthermore, profibrogenic signaling is also activated in HSCs leading to liver fibrosis (90). Consistently, Kornek et al., revealed experimental data for the presence of EVs carrying $\mathrm{CD}^{+}$and $\mathrm{CD}^{+} \mathrm{T}$ cells, NK cells in the plasma of NAFLD patients (91). Adipocyte-derived EVs exert a crucial role in augmenting insulin resistance and triggering hepatic inflammation via MCP-1 and IL-6 (92). Notably, Thomou et al., have provided evidence that adipose tissue serves as a major source of EVs carrying micro RNA using mice lacking dicer in adipose tissue (93).

Currently, NAFLD has no efficient technique of diagnosis for staging the disease except for the highly invasive gold standard technique of liver biopsy. Multiple non-invasive techniques have been practiced to assess NAFLD severity such as analyzing serum levels of liver enzymes, ultrasound-based elastography, and magnetic resonance imaging (MRI) (94). However, these techniques lack proper efficacy and precision in early diagnosis, severity, and grading of disease stage (95). The implication of EVs in the pathobiology of different stages in NAFLD spectra has made it a potent biomarker in NAFLD diagnosis $(15,96)$. Due to their availability in biological fluids such as blood and ease of intercellular communication, EVs are proposed to be an excellent non-invasive liquid biomarker referred to as "liquid biopsy". There are several EV biomarkers (protein-based, lipid-based, or nucleic acid-based EV biomarkers) that have been documented and it is known that the abundance and molecular composition of these circulating EVs vary and signify different disease conditions which would help in improving the current status of diagnosis of NAFLD (97-99). Importantly, EVs have emerged as a therapeutic tool in treating NAFLD due to their ability in stable delivery of drugs, miRNA, siRNA, or other cargoes and easy uptake by the liver cells paving the path to hope towards EV-based therapy. Additionally, studies have also demonstrated EVs as a potential therapeutic target as inhibition of EV-mediated pathological processes can ameliorate disease progression in NAFLD $(78,89)$.

\section{CONCLUSIONS}

NAFLD is a multifactorial complex diseased condition caused by multiple parallel hits and covers a wide spectrum of liver damage. Accumulation of toxic lipid species linked with inter-organ crosstalk of adipose tissue-liver axis and gut-liver axis contributes to the activation of the innate immune system and inflammatory signals through several cytokines and chemokines. Developed over the last decades is the idea of crosstalk in the pathogenesis of NASH via circulating extracellular vesicles. Many studies conducted over this period have revealed the intricate role of EVs in driving several features of NASH such as inflammation, innate immune response, profibrogenic activation most of which are rooted in lipotoxicity. Thus, EVs are important molecular entities involved as mediators in cellcell and organ-organ communication contributing to liver physiology and pathophysiology. 
Exploiting the attractive and easy circulating nature of EVs, several of them have been characterized as a potential biomarker candidate. Given the significance, upon further critical evaluation of these EVs, it is likely to emerge as a promising biomarker for easy diagnosis, assessing the severity and stages of the disease. However, there still remains an uphill battle in terms of characterizing and investigating the EVs due to their heterogeneity and differential expression in various diseases. Similarly, the feasibility of blockade of EV secretion as a therapeutic strategy is far beyond implication, as it demands a further understanding of the role of EVs in normal physiology. Active research and collaboration between scientists and physicians would pay a way for the successful transition of current EV knowledge from bench to bedside. In conclusion, the comprehensive investigation leading to a better understanding of the regulation and mechanisms of EV biogenesis and secretion and functional analysis of its

\section{REFERENCES}

1. Loomba R, Sanyal AJ. The global NAFLD epidemic. Nat Rev Gastroenterol Hepatol (2013) 10(11):686-90. doi: 10.1038/nrgastro.2013.171

2. Farrell GC, Larter CZ. Nonalcoholic fatty liver disease: from steatosis to cirrhosis. Hepatology (2006) 43(2 Suppl 1):S99-S112. doi: 10.1002/hep.20973

3. Byrne CD, Targher G. NAFLD: a multisystem disease. J Hepatol (2015) 62(1 Suppl):S47-64. doi: 10.1016/j.jhep.2014.12.012

4. Sanyal AJ. Past, present and future perspectives in nonalcoholic fatty liver disease. Nat Rev Gastroenterol Hepatol (2019) 16(6):377-86. doi: 10.1038/ s41575-019-0144-8

5. Younossi ZM. Non-alcoholic fatty liver disease - A global public health perspective. J Hepatol (2019) 70(3):531-44. doi: 10.1016/j.jhep.2018.10.033

6. Estes C, Razavi H, Loomba R, Younossi Z, Sanyal AJ. Modeling the epidemic of nonalcoholic fatty liver disease demonstrates an exponential increase in burden of disease. Hepatology (2018) 67(1):123-33. doi: 10.1002/hep.29466

7. Estes C, Anstee QM, Arias-Loste MT, Bantel H, Bellentani S, Caballeria J, et al. Modeling NAFLD disease burden in China, France, Germany, Italy, Japan, Spain, United Kingdom, and United States for the period 2016-2030. J Hepatol (2018) 69(4):896-04. doi: 10.1016/j.jhep.2018.05.036

8. Younossi ZM, Koenig AB, Abdelatif D, Fazel Y, Henry L, Wymer M. Global epidemiology of nonalcoholic fatty liver disease-Meta-analytic assessment of prevalence, incidence, and outcomes. Hepatology (2016) 64(1):73-84. doi: $10.1002 /$ hep. 28431

9. Cotter TG, Rinella M. Nonalcoholic Fatty Liver Disease 2020: The State of the Disease. Gastroenterology (2020) 158(7):1851-64. doi: 10.1053/j.gastro.2020.01.052

10. Buzzetti E, Pinzani M, Tsochatzis EA. The multiple-hit pathogenesis of nonalcoholic fatty liver disease (NAFLD). Metabolism (2016) 65(8):1038-48. doi: 10.1016/j.metabol.2015.12.012

11. Friedman SL, Neuschwander-Tetri BA, Rinella M, Sanyal AJ. Mechanisms of NAFLD development and therapeutic strategies. Nat Med (2018) 24(7):90822. doi: 10.1038/s41591-018-0104-9

12. Harmon RC, Tiniakos DG, Argo CK. Inflammation in nonalcoholic steatohepatitis. Expert Rev Gastroenterol Hepatol (2011) 5(2):189-200. doi: 10.1586/egh.11.21

13. Ban LA, Shackel NA, McLennan SV. Extracellular Vesicles: A New Frontier in Biomarker Discovery for Non-Alcoholic Fatty Liver Disease. Int J Mol Sci (2016) 17(3):376. doi: 10.3390/ijms17030376

14. Cai S, Cheng X, Pan X, Li J. Emerging role of exosomes in liver physiology and pathology. Hepatol Res (2017) 47(2):194-203. doi: 10.1111/hepr.12794

15. Garcia-Martinez I, Alen R, Rada P, Valverde AM. Insights Into Extracellular Vesicles as Biomarker of NAFLD Pathogenesis. Front Med (Lausanne) (2020) 7:395:395. doi: 10.3389/fmed.2020.00395 associated cargoes would aid in filling the much-needed lacuna in the diagnosis and therapy of NAFLD.

\section{AUTHOR CONTRIBUTIONS}

AS and DK devised and wrote the manuscript. DiS and DK made the figures. PS and DeS provided input and edited the manuscript. All authors contributed to the article and approved the submitted version.

\section{FUNDING}

This study was supported by Ramalingaswami Re-entry Fellowship to DPK from the Department of Biotechnology (DBT), Government of India.
16. Sato K, Meng F, Glaser S, Alpini G. Exosomes in liver pathology. J Hepatol (2016) 65(1):213-21. doi: 10.1016/j.jhep.2016.03.004

17. Neuschwander-Tetri BA. Hepatic lipotoxicity and the pathogenesis of nonalcoholic steatohepatitis: the central role of nontriglyceride fatty acid metabolites. Hepatology (2010) 52(2):774-88. doi: 10.1002/hep.23719

18. Cheung O, Sanyal AJ. Abnormalities of lipid metabolism in nonalcoholic fatty liver disease. Semin Liver Dis (2008) 28(4):351-59. doi: 10.1055/s-0028-1091979

19. Mendez-Sanchez N, Cruz-Ramon VC, Ramirez-Perez OL, Hwang JP, Barranco-Fragoso B, Cordova-Gallardo J. New Aspects of Lipotoxicity in Nonalcoholic Steatohepatitis. Int J Mol Sci (2018) 19(7):2034. doi: 10.3390/ ijms19072034

20. Yamaguchi K, Yang L, McCall S, Huang J, Yu XX, Pandey SK. Inhibiting triglyceride synthesis improves hepatic steatosis but exacerbates liver damage and fibrosis in obese mice with nonalcoholic steatohepatitis. Hepatology (2007) 45(6):1366-74. doi: 10.1002/hep.21655

21. Musso G, Cassader M, Paschetta E, Gambino R. Bioactive Lipid Species and Metabolic Pathways in Progression and Resolution of Nonalcoholic Steatohepatitis. Gastroenterology (2018) 155(2):282-302. doi: 10.1053/ j.gastro.2018.06.031

22. Akazawa Y, Cazanave S, Mott JL, Elmi N, Bronk SF, Kohno S, et al. Palmitoleate attenuates palmitate-induced Bim and PUMA up-regulation and hepatocyte lipoapoptosis. J Hepatol (2010) 52(4):586-93. doi: 10.1016/ j.jhep.2010.01.003

23. Parthasarathy G, Revelo X, Malhi H. Pathogenesis of Nonalcoholic Steatohepatitis: An Overview. Hepatol Commun (2020) 4(4):478-92. doi: 10.1002/hep4.1479

24. Puri P, Mirshahi F, Cheung O, Natarajan R, Maher JW, Kellum JM, et al. Activation and dysregulation of the unfolded protein response in nonalcoholic fatty liver disease. Gastroenterology (2008) 134(2):568-76. doi: 10.1053/ j.gastro.2007.10.039

25. Han J, Kaufman RJ. The role of ER stress in lipid metabolism and lipotoxicity. J Lipid Res (2016) 57(8):1329-38. doi: 10.1194/jlr.R067595

26. Guy CD, Suzuki A, Zdanowicz M, Abdelmalek MF, Burchette J, Unalp A, et al. NASH CRN. Hedgehog pathway activation parallels histologic severity of injury and fibrosis in human nonalcoholic fatty liver disease. Hepatology (2012) 55(6):1711-21. doi: 10.1002/hep.25559

27. Wree A, Eguchi A, McGeough MD, Pena CA, Johnson CD, Canbay A, et al. NLRP3 inflammasome activation results in hepatocyte pyroptosis, liver inflammation, and fibrosis in mice. Hepatology (2014) 59(3):898-10. doi: 10.1002/hep.26592

28. Guo J, Friedman SL. Toll-like receptor 4 signaling in liver injury and hepatic fibrogenesis. Fibrogenesis Tissue Repair (2010) 3:21. doi: 10.1186/1755-15363-21 
29. Cazanave SC, Mott JL, Bronk SF, Werneburg NW, Fingas CD, Meng XW, et al. Death receptor 5 signaling promotes hepatocyte lipoapoptosis. J Biol Chem (2011) 286(45):39336-48. doi: 10.1074/jbc.M111.280420

30. Masarone M, Rosato V, Dallio M, Gravina AG, Aglitti A, Loguercio C, et al. Role of Oxidative Stress in Pathophysiology of Nonalcoholic Fatty Liver Disease. Oxid Med Cell Longev (2018) 11(2018):9547613. doi: 10.1155/2018/ 9547613

31. Schoeler M, Caesar R. Dietary lipids, gut microbiota and lipid metabolism. Rev Endocr Metab Disord (2019) 20(4):461-72. doi: 10.1007/s11154-019-09512-0

32. Youssef OA, Safran SA, Nakamura T, Nix DA, Hotamisligil GS, Bass BL. Potential role for snoRNAs in PKR activation during metabolic stress. Proc Natl Acad Sci U S A (2015) 112(16):5023-8. doi: 10.1073/pnas.1424044112

33. Marra F, Svegliati-Baroni G. Lipotoxicity and the gut-liver axis in NASH pathogenesis. J Hepatol (2018) 68(2):280-95. doi: 10.1016/j.jhep.2017.11.014

34. Caligiuri A, Gentilini A, Marra F. Molecular Pathogenesis of NASH. Int J Mol Sci (2016) 17(9):1575. doi: 10.3390/ijms 17091575

35. Carlos FF, Trigueiro APR, Sousa AVVP, Andrade KS, Henriques MSM. Role of cytokines in pathogenesis and progression of nonalcoholic steatohepatitis. Gastroenterol Hepatol Open Access (2019) 10(3):126-30. doi: 10.15406/ ghoa.2019.10.00370

36. Braunersreuther V, Viviani GL, Mach F, Montecucco F. Role of cytokines and chemokines in non-alcoholic fatty liver disease. World J Gastroenterol (2012) 18(8):727-35. doi: 10.3748/wjg.v18.i8.727

37. Lambertucci F, Arboatti A, Sedlmeier MG, Motiño O, Alvarez ML, Ceballos MP, et al. Disruption of tumor necrosis factor alpha receptor 1 signaling accelerates NAFLD progression in mice upon a high-fat diet. J Nutr Biochem (2018) 58:17-27. doi: 10.1016/j.jnutbio.2018.04.013

38. Klein C, Wüstefeld T, Assmus U, Roskams T, Rose-John S, Müller M, et al. The IL-6-gp130-STAT3 pathway in hepatocytes triggers liver protection in T cell-mediated liver injury. J Clin Invest (2005) 115(4):860-9. doi: 10.1172/ JCI23640

39. Nov O, Shapiro H, Ovadia H, Tarnovscki T, Dvir I, Shemesh E, et al. Interleukin-1 $\beta$ Regulates Fat-Liver Crosstalk in Obesity by Auto-Paracrine Modulation of Adipose Tissue Inflammation and Expandability. PloS One (2013) 8(1):e53626. doi: 10.1371/journal.pone.0053626

40. Niederreiter L, Tilg H. Cytokines and fatty liver diseases. Liver Res (2018) 2 (1):14-20. doi: 10.1016/j.livres.2018.03.003

41. den Boer MA, Voshol PJ, Schr öder-van der Elst JP, Korsh- eninnikova E, Ouwens DM, et al. Endogenous interleukin-10 protects against hepatic steatosis but does not improve insulin sensitivity during high-fat feeding in mice. Endocrinology (2006) 147:4553-8. doi: 10.1210/en.2006-0417

42. Cintra DE, Pauli JR, Araújo EP, Moraes JC, de Souza CT, Milanski M, et al. Interleukin-10 is a protective factor against diet-induced insulin resistance in liver. J Hepatol (2008) 48(4):628-37. doi: 10.1016/j.jhep.2007.12.017

43. Wulster-Radcliffe MC, Ajuwon KM, Wang J, Christian JA, Spurlock ME. Adiponectin differentially regulates cytokines in porcine macrophages. Biochem Biophys Res Commun (2004) 316(3):924-9. doi: 10.1016/ j.bbrc.2004.02.130

44. Xu L, Kitade H, Ni Y, Ota T. Roles of Chemokines and Chemokine Receptors in Obesity-Associated Insulin Resistance and Nonalcoholic Fatty Liver Disease. Biomolecules (2015) 5(3):1563-79. doi: 10.3390/biom5031563

45. Chen W, Zhang J, Fan HN, Zhu JS. Function and therapeutic advances of chemokine and its receptor in nonalcoholic fatty liver disease. Therap Adv Gastroenterol (2018) 11:1756284818815184. doi: 10.1177/1756284818815184

46. Milosevic I, Vujovic A, Barac A, Djelic M, Korac M, Radovanovic Spurnic A, et al. Gut-Liver Axis, Gut Microbiota, and Its Modulation in the Management of Liver Diseases: A Review of the Literature. Int J Mol Sci (2019) 20(2):395. doi: 10.3390/ijms20020395

47. Leung C, Rivera L, Furness JB, Angus PW. The role of the gut microbiota in NAFLD. Nat Rev Gastroenterol Hepatol (2016) 13(7):412-25. doi: 10.1038/ nrgastro. 2016.85

48. Aragonès G, González-García S, Aguilar C, Richart C, Auguet T. Gut Microbiota-Derived Mediators as Potential Markers in Nonalcoholic Fatty Liver Disease. BioMed Res Int (2019) 2019:8507583. doi: 10.1155/2019/ 8507583

49. Saltzman ET, Palacios T, Thomsen M, Vitetta L. Intestinal Microbiome Shifts, Dysbiosis, Inflammation, and Non-alcoholic Fatty Liver Disease. Front Microbiol (2018) 9:61:61. doi: 10.3389/fmicb.2018.00061
50. Wang R, Li H, Yang X, Xue X, Deng L, Shen J, et al. Genetically Obese Human Gut Microbiota Induces Liver Steatosis in Germ-Free Mice Fed on Normal Diet. Front Microbiol (2018) 9:1602:1602. doi: 10.3389/fmicb.2018.01602

51. Le Roy T, Llopis M, Lepage P, Bruneau A, Rabot S, Bevilacqua C, et al. Intestinal microbiota determines development of non-alcoholic fatty liver disease in mice. Gut (2013) 62(12):1787-94. doi: 10.1136/gutjnl-2012-303816

52. Bibbò S, Ianiro G, Dore MP, Simonelli C, Newton EE, Cammarota G. Gut Microbiota as a Driver of Inflammation in Nonalcoholic Fatty Liver Disease. Mediators Inflamm (2018) 31:9321643. doi: 10.1155/2018/9321643

53. Henao-Mejia J, Elinav E, Jin C, Hao L, Mehal WZ, Strowig T, et al. Inflammasome-mediated dysbiosis regulates progression of NAFLD and obesity. Nature (2012) 482(7384):179-85. doi: 10.1038/nature10809

54. Mridha AR, Wree A, Robertson AAB, Yeh MM, Johnson CD, Van Rooyen $\mathrm{DM}$, et al. NLRP3 inflammasome blockade reduces liver inflammation and fibrosis in experimental NASH in mice. J Hepatol (2017) 66(5):1037-46. doi: 10.1016/j.jhep.2017.01.022

55. Akers JC, Gonda D, Kim R, Carter BS, Chen CC. Biogenesis of extracellular vesicles $(\mathrm{EV})$ : exosomes, microvesicles, retrovirus-like vesicles, and apoptotic bodies. J Neurooncol (2013) 113(1):1-11. doi: 10.1007/s11060-013-1084-8

56. Harding C, Heuser J, Stahl P. Receptor-mediated endocytosis of transferrin and recycling of the transferrin receptor in rat reticulocytes. J Cell Biol (1983) 97(2):329-39. doi: 10.1083/jcb.97.2.329

57. Pan BT, Johnstone RM. Fate of the transferrin receptor during maturation of sheep reticulocytes in vitro: selective externalization of the receptor. Cell (1983) 33(3):967-78. doi: 10.1016/0092-8674(83)90040-5

58. Lötvall J, Hill AF, Hochberg F, Buzás EI, Di Vizio D, Gardiner C, et al. Minimal experimental requirements for definition of extracellular vesicles and their functions: a position statement from the International Society for Extracellular Vesicles. J Extracell Vesicles (2014) 3:26913. doi: 10.3402/ jev.v3.26913

59. Théry C, Zitvogel L, Amigorena S. Exosomes: composition, biogenesis and function. Nat Rev Immunol (2002) 2(8):569-79. doi: 10.1038/nri855

60. Escola JM, Kleijmeer MJ, Stoorvogel W, Griffith JM, Yoshie O, Geuze HJ. Selective enrichment of tetraspan proteins on the internal vesicles of multivesicular endosomes and on exosomes secreted by human Blymphocytes. J Biol Chem (1998) 273(32):20121-7. doi: 10.1074/ jbc.273.32.20121

61. Abels ER, Breakefield XO. Introduction to Extracellular Vesicles: Biogenesis, RNA Cargo Selection, Content, Release, and Uptake. Cell Mol Neurobiol (2016) 36(3):301-12. doi: 10.1007/s10571-016-0366-Z

62. Raiborg C, Stenmark H. The ESCRT machinery in endosomal sorting of ubiquitylated membrane proteins. Nature (2009) 458(7237):445-52. doi: $10.1038 /$ nature07961

63. Stuffers S, Sem Wegner C, Stenmark H, Brech A. Multivesicular endosome biogenesis in the absence of ESCRTs. Traffic (2009) 10(7):925-37. doi: 10.1111/j.1600-0854.2009.00920.x

64. Raposo G, Stoorvogel W. Extracellular vesicles: exosomes, microvesicles, and friends. J Cell Biol (2013) 200(4):373-83. doi: 10.1083/jcb.201211138

65. Yáñez-Mó M, Siljander PR, Andreu Z, Zavec AB, Borràs FE, Buzas EI, et al. Biological properties of extracellular vesicles and their physiological functions. J Extracell Vesicles (2015) 4:27066. doi: 10.3402/jev.v4.27066

66. Morán L, Cubero FJ. Extracellular vesicles in liver disease and beyond. World J Gastroenterol (2018) 24(40):4519-26. doi: 10.3748/wjg.v24.i40.4519

67. Povero D, Eguchi A, Li H, Johnson CD, Papouchado BG, Wree A, et al. Circulating extracellular vesicles with specific proteome and liver microRNAs are potential biomarkers for liver injury in experimental fatty liver disease. PloS One (2014) 9(12):e113651. doi: 10.1371/journal.pone.0113651

68. Malhi H, Barreyro FJ, Isomoto H, Bronk SF, Gores GJ. Free fatty acids sensitise hepatocytes to TRAIL mediated cytotoxicity. Gut (2007) 56(8):112431. doi: 10.1136/gut.2006.118059

69. Newman LA, Sorich MJ, Rowland A. Role of Extracellular Vesicles in the Pathophysiology, Diagnosis and Tracking of Non-Alcoholic Fatty Liver Disease. J Clin Med (2020) 9(7):2032. doi: 10.3390/jcm9072032

70. Devhare PB, Ray RB. Extracellular vesicles: Novel mediator for cell to cell communications in liver pathogenesis. Mol Aspects Med (2018) 60:115-22. doi: 10.1016/j.mam.2017.11.001

71. Banales JM, Feldstein AE, Sänger H, Lukacs-Kornek V, Szabo G, Kornek M. Extracellular Vesicles in Liver Diseases: Meeting Report from the 
International Liver Congress 2018. Hepatol Commun (2019) 3(2):305-15. doi: 10.1002/hep4.1300

72. Overi D, Carpino G, Franchitto A, Onori P, Gaudio E. Hepatocyte Injury and Hepatic Stem Cell Niche in the Progression of Non-Alcoholic Steatohepatitis. Cells (2020) 9(3):590. doi: 10.3390/cells9030590

73. Kakazu E, Mauer AS, Yin M, Malhi H. Hepatocytes release ceramide-enriched pro-inflammatory extracellular vesicles in an IRE1 $\alpha$-dependent manner. $J$ Lipid Res (2016) 57(2):233-45. doi: 10.1194/jlr.M063412

74. Hirsova P, Gores GJ. Death Receptor-Mediated Cell Death and Proinflammatory Signaling in Nonalcoholic Steatohepatitis. Cell Mol Gastroenterol Hepatol (2015) 1(1):17-27. doi: 10.1016/j.jcmgh.2014.11.005

75. Hirsova P, Ibrahim SH, Gores GJ, Malhi H. Lipotoxic lethal and sublethal stress signaling in hepatocytes: relevance to NASH pathogenesis [published correction appears in J Lipid Res. 2017 Jan;58(1):299. [corrected to Ibrahim SH]]. J Lipid Res (2016) 57(10):1758-70. doi: 10.1194/jlr.R066357

76. Schattenberg JM, Lee MS. Extracellular Vesicles as Messengers Between Hepatocytes and Macrophages in Nonalcoholic Steatohepatitis. Gastroenterology (2016) 150(4):815-18. doi: 10.1053/j.gastro.2016.02.064

77. Hirsova P, Ibrahim SH, Krishnan A, Verma VK, Bronk SF, Werneburg NW, et al. Lipid-Induced Signaling Causes Release of Inflammatory Extracellular Vesicles From Hepatocytes. Gastroenterology (2016) 150(4):956-67. doi: 10.1053/j.gastro.2015.12.037

78. Ibrahim SH, Hirsova P, Tomita K, Bronk SF, Werneburg NW, Harrison SA, et al. Mixed lineage kinase 3 mediates release of C-X-C motif ligand 10bearing chemotactic extracellular vesicles from lipotoxic hepatocytes. Hepatology (2016) 63(3):731-44. doi: 10.1002/hep.28252

79. Garcia-Martinez I, Santoro N, Chen Y, Hoque R, Ouyang X, Caprio S, et al. Hepatocyte mitochondrial DNA drives nonalcoholic steatohepatitis by activation of TLR9. J Clin Invest (2016) 126(3):859-64. doi: 10.1172/JCI83885

80. Cannito S, Morello E, Bocca C, Foglia B, Benetti E, Novo E, et al. Microvesicles released from fat-laden cells promote activation of hepatocellular NLRP3 inflammasome: A pro-inflammatory link between lipotoxicity and nonalcoholic steatohepatitis. PloS One (2017) 12(3):e0172575. doi: 10.1371/ journal.pone. 0172575

81. Guo Q, Furuta K, Lucien F, Gutierrez Sanchez LH, Hirsova P, Krishnan A, et al. Integrin $\beta_{1}$-enriched extracellular vesicles mediate monocyte adhesion and promote liver inflammation in murine NASH. J Hepatol (2019) 71 (6):1193-05. doi: 10.1016/j.jhep.2019.07.019

82. Jiang F, Chen Q, Wang W, Ling Y, Yan Y, Xia P. Hepatocyte-derived extracellular vesicles promote endothelial inflammation and atherogenesis via microRNA-1. J Hepatol (2020) 72(1):156-66. doi: 10.1016/ j.jhep.2019.09.014

83. Zhao Y, Zhao MF, Jiang S, Wu J, Liu J, Yuan XW, et al. Liver governs adipose remodelling via extracellular vesicles in response to lipid overload. Nat Commun (2020) 11(1):719. doi: 10.1038/s41467-020-14450-6

84. Liu XL, Pan Q, Cao HX, Xin FZ, Zhao ZH, Yang RX, et al. Lipotoxic Hepatocyte-Derived Exosomal MicroRNA 192-5p Activates Macrophages Through Rictor/Akt/Forkhead Box Transcription Factor O1 Signaling in Nonalcoholic Fatty Liver Disease. Hepatology (2020) 72(2):454-69. doi: 10.1002/hep.31050

85. Povero D, Eguchi A, Niesman IR, Andronikou N, de Mollerat du Jeu X, Mutya A, et al. Lipid-induced toxicity stimulates hepatocytes to release angiogenic microparticles that require Vanin-1 for uptake by endothelial cells. Sci Signal (2013) 6(296):ra88. doi: 10.1126/scisignal.2004512

86. Lee YS, Kim SY, Ko E, Lee JH, Yi HS, Yoo YJ, et al. Exosomes derived from palmitic acid-treated hepatocytes induce fibrotic activation of hepatic stellate cells. Sci Rep (2017) 7(1):3710. doi: 10.1038/s41598-017-03389-2
87. Becker PP, Rau M, Schmitt J, Malsch C, Hammer C, Bantel H, et al. Performance of Serum microRNAs -122, -192 and -21 as Biomarkers in Patients with Non-Alcoholic Steatohepatitis. PloS One (2015) 10(11): e0142661. doi: 10.1371/journal.pone.0142661

88. Eguchi A, Feldstein AE. Extracellular vesicles in non-alcoholic and alcoholic fatty liver diseases. Liver Res (2018) 2(1):30-4. doi: 10.1016/ j.livres.2018.01.001

89. Wang R, Ding Q, Yaqoob U, de Assuncao TM, Verma VK, Hirsova P, et al. Exosome Adherence and Internalization by Hepatic Stellate Cells Triggers Sphingosine 1-Phosphate-dependent Migration. J Biol Chem (2015) 290 (52):30684-96. doi: 10.1074/jbc.M115.671735

90. Charrier A, Chen R, Chen L, Kemper S, Hattori T, Takigawa M, et al. Exosomes mediate intercellular transfer of pro-fibrogenic connective tissue growth factor (CCN2) between hepatic stellate cells, the principal fibrotic cells in the liver. Surgery (2014) 156(3):548-55. doi: 10.1016/j.surg.2014.04.014

91. Kornek M, Popov Y, Libermann TA, Afdhal NH, Schuppan D. Human T cell microparticles circulate in blood of hepatitis patients and induce fibrolytic activation of hepatic stellate cells. Hepatology (2011) 53(1):230-42. doi: 10.1002/hep.23999

92. Kranendonk ME, Visseren FL, van Herwaarden JA, Nolte-'t Hoen EN, de Jager W, Wauben MH, et al. Effect of extracellular vesicles of human adipose tissue on insulin signaling in liver and muscle cells. Obesity (Silver Spring) (2014) 22(10):2216-23. doi: 10.1002/oby.20847

93. Thomou T, Mori MA, Dreyfuss JM, Konishi M, Sakaguchi M, Wolfrum C, et al. Adipose-derived circulating miRNAs regulate gene expression in other tissues. Nature (2017) 542(7642):450-55. doi: 10.1038/nature21365

94. Wong VW, Adams LA, de Lédinghen V, Wong GL, Sookoian S. Noninvasive biomarkers in NAFLD and NASH - current progress and future promise. Nat Rev Gastroenterol Hepatol (2018) 15(8):461-78. doi: 10.1038/s41575-0180014-9

95. Younossi ZM, Loomba R, Rinella ME, Bugianesi E, Marchesini G, Neuschwander-Tetri BA, et al. Current and future therapeutic regimens for nonalcoholic fatty liver disease and nonalcoholic steatohepatitis. Hepatology (2018) 68(1):361-71. doi: 10.1002/hep.29724

96. Szabo G, Momen-Heravi F. Extracellular vesicles in liver disease and potential as biomarkers and therapeutic targets. Nat Rev Gastroenterol Hepatol (2017) 14(8):455-66. doi: 10.1038/nrgastro.2017.71

97. Mann J, Reeves HL, Feldstein AE. Liquid biopsy for liver diseases. Gut (2018) 67(12):2204-12. doi: 10.1136/gutjnl-2017-315846

98. Zhou B, Xu K, Zheng X, Chen T, Wang J, Song Y, et al. Application of exosomes as liquid biopsy in clinical diagnosis. Signal Transduct Target Ther (2020) 5(1):144. doi: 10.1038/s41392-020-00258-9

99. Urban SK, Mocan T, Sänger H, Lukacs-Kornek V, Kornek M. Extracellular Vesicles in Liver Diseases: Diagnostic, Prognostic, and Therapeutic Application. Semin Liver Dis (2019) 39(1):70-7. doi: 10.1055/s-0038-1676122

Conflict of Interest: The authors declare that the research was conducted in the absence of any commercial or financial relationships that could be construed as a potential conflict of interest.

Copyright (c) 2021 Srinivas, Suresh, Santhekadur, Suvarna and Kumar. This is an open-access article distributed under the terms of the Creative Commons Attribution License (CC BY). The use, distribution or reproduction in other forums is permitted, provided the original author(s) and the copyright owner(s) are credited and that the original publication in this journal is cited, in accordance with accepted academic practice. No use, distribution or reproduction is permitted which does not comply with these terms. 